\title{
Perovskite Quantum Dots Modeled Using ab Initio and Replica Exchange Molecular Dynamics
}

\author{
Andrei Buin, Riccardo Comin, Alexander H. Ip, and \\ Edward H. Sargent
}

\author{
Version Post-Print/Accepted Manuscript \\ Citation Buin, A., Comin, R., Ip, A., \& Sargent, E. (2015). Perovskite Quantum \\ (published version) Dots Modeled Using ab Initio and Replica Exchange Molecular \\ Dynamics. J. Phys. Chem. C, 119(24), 13965-13971. \\ http://dx.doi.org/10.1021/acs.jpcc.5b03613
}

Publisher's Statement This document is the Accepted Manuscript version of a Published Work that appeared in final form in The Journal of Physical Chemistry $C$, copyright (C2015 American Chemical Society after peer review and technical editing by the publisher. To access the final edited and published work see http://dx.doi.org/10.1021/acs.jpcc.5b03613.

\section{How to cite TSpace items}

Always cite the published version, so the author(s) will receive recognition through services that track citation counts, e.g. Scopus. If you need to cite the page number of the TSpace version (original manuscript or accepted manuscript) because you cannot access the published version, then cite the TSpace version in addition to the published version using the permanent URI (handle) found on the record page. 
This document is confidential and is proprietary to the American Chemical Society and its authors. Do not copy or disclose without written permission. If you have received this item in error, notify the sender and delete all copies.

\section{Perovskite Quantum Dots Modeled Using Ab Initio and Replica Exchange Molecular Dynamics}

\begin{tabular}{|r|l|}
\hline Journal: & The Journal of Physical Chemistry \\
\hline Manuscript ID & jp-2015-036136.R1 \\
\hline Manuscript Type: & Article \\
\hline Date Submitted by the Author: & $29-$ May-2015 \\
\hline & $\begin{array}{l}\text { Buin, Andrei; University of Toronto, ECE } \\
\text { Comin, Riccardo; University of Toronto, ECE } \\
\text { Sargent, Edward; University of Ond Toronto, Electrical and Computer Engineering } \\
\text { Engineering; }\end{array}$ \\
\hline
\end{tabular}

\section{SCHOLARONE \\ Manuscripts}




\author{
Andrei Buin, Riccardo Comin, Alexander H. Ip, and Edward H. Sargent* \\ Department of Electrical and Computer Engineering, University of Toronto, Toronto, \\ Canada, ON M5S 3G4 \\ E-mail: ted.sargent@utoronto.ca \\ Phone: +1 (416)946 5051
}

\begin{abstract}
Organometal halide perovskites have recently attracted tremendous attention both at the experimental and theoretical levels. Much of this work has been dedicated to bulk material studies, yet recent experimental work has shown the formation of highly efficient quantum-confined nanocrystals with tunable band edges. Here we investigate perovskite quantum dots from theory, predicting an upper bound of the Bohr radius of $45 \AA$ that agrees well with literature values. When the quantum dots are stoichiometric, they are trap-free and have nearly symmetric contributions to confinement from the valence and conduction bands. We further show that surfaceassociated conduction bandedge states in perovskite nanocrystals lie below the bulk states, which could explain the difference in Urbach tails between mesoporous and planar perovskite films. In addition to conventional molecular dynamics (MD), we implement an enhanced phase-space sampling algorithm, replica exchange molecular dynamics (REMD). We find that, in simulating methylammonium orientation and global minima, REMD outperforms conventional MD. To the best of our knowledge, this is the first REMD implementation for realistic-sized systems in the realm of DFT calculations.
\end{abstract}

Keywords: Perovskite, Quantum dots, Electronic structure, DFT

The authors declare no competing financial interest.

${ }^{*}$ To whom correspondence should be addressed 
The perovskite photovoltaic field has blossomed in the past few years due to facile solution processing and impressive power conversion efficiencies. ${ }^{1,2}$ To date, the majority of the experimental and theoretical work ${ }^{3-16}$ has been dedicated to the bulk properties of the perovskite material. There are two main architectures employed for perovskite solar cell fabrication, namely mesoporous ${ }^{1,2}$ and planar. ${ }^{17}$ Mesoscopic perovskite structures are typically formed using mesoporous $\mathrm{TiO}_{2}$ electrodes, with pore sizes being on the order of a few tens of nanometers. Strictly speaking the perovskite is already nanocrystalline; however, due to the relatively large grain size the effects of quantum confinement are lost. Recently, experimental work ${ }^{18,19}$ appeared regarding the fabrication of quantum dots (QDs), in which confinement is preserved, thus giving an opportunity for bandgap tuning for optoelectronic applications. In order to gain a deeper understanding of these interesting materials, we perform calculations using rigorous density functional theory (DFT) of quantum dots with various stoichiometries and sizes (240-768 atoms) built from cubic methylammonium (MA) lead iodide perovskite $\left(\mathrm{MAPbI}_{3}\right)$.

It is known that bulk $\mathrm{MAPbI}_{3}$ possesses the tetragonal structure as the most stable form at room temperature; however, at the nanoscale the stability of the bulk phase might be altered due to surface energy considerations and synthesis routes. Indeed, recent studies ${ }^{20-22}$ indicate that mixed halide synthesis in addition to nanoscale phenomena can produce $\mathrm{MAPbI}_{3}$ samples with the cubic symmetry. For the mixed halide synthesis route, it was partially ascribed to the presence the $\mathrm{Cl}$ which lowers the transition temperature of the tetragonal-cubic phase. The effect of the surface energy term at the nanoscale has been demonstrated in the case of nanoscale ${ }^{23} \mathrm{TiO}_{2}$ particles, and indeed in recent work by Protesescu et al. ${ }^{18}$ such phenomena has been demonstrated for the example of $\mathrm{CsPbI}_{3}$ quantum dots in which they crystallize into the cubic crystal structure. This justifies usage of the cubic phase of $\mathrm{MAPbI}_{3}$ while simplifying the DFT calculations.

In order to demonstrate the effect of quantum confinement we have performed MD calculations of various sizes of stoichiometric quantum dots (QDs). The calculations were run at $100 \mathrm{~K}$ rather than 300 $\mathrm{K}$ because at $300 \mathrm{~K}$ the standard deviation of bandgap becomes comparable to the amount of quantum confinement itself. As for the off-stoichiometric QDs, we have performed MD at various temperatures and tried various sizes with various stoichiometries (see Supporting Information Figure S1). However, we could not obtain trap-free QDs, which is consistent with previously reported trap introduction due 
to violation of the bulk stoichiometry. ${ }^{24,25}$ We define trap-free as having no electronic states in the gap region greater than $10 \mathrm{kT}$ from any of the bandedges while subgap states are defined as tails below conduction band minimum or above valence band maximum within $10 \mathrm{kT}$.

Figure 1(a) shows variation of the bandgap of stoichiometric QDs vs. particle radii where confinement is apparent and no emerging in-gap states are seen. This result is in good agreement with recent experimental work, ${ }^{18}$ where $\mathrm{CsPbI}_{3}$ nanocrystals with near bulk stoichiometry and with very narrow emission lines have been fabricated. Within the DFT realm it is not possible to account for the exciton phenomena; however, it is still possible to give a rough estimate to the upper bound of the Bohr radius for excitons. The excitonic Bohr radius is defined as a cross-over point between the strong confinement regime, where confinement effects dominate, and an intermediate to weak confinement regime where excitonic Coulomb and correlation energies dominate over the confinement energies. Given that exciton binding energy in bulk $\mathrm{MAPbI}_{3}$ varies between $35-50 \mathrm{meV}^{26,27}$ and that exciton binding energy is 48 $\mathrm{meV}^{28}$ at a Bohr radius of $25 \AA$, one can assume that exciton binding energy remains constant beyond Bohr radius. The fit for the bandgap variation in Figure 1(a) can be written as:

$$
E_{g}=E_{g}^{b u l k}+\frac{b}{r^{2}}
$$

where $b\left(\approx 100 \mathrm{eV}^{*} \AA^{2}\right)$ is a constant which absorbs all the effects of the confining potential and effective electron and hole masses, ${ }^{29}$ and $\mathrm{E}_{g}^{b u l k}$ is the bulk bandgap of the $\mathrm{MAPbI}_{3}$. Given the exciton binding energy one can get an estimate of the upper bound on the Bohr radius, which is given by:

$$
\left(r_{B}^{<=}\right)^{-1}=\sqrt{\frac{E_{b}}{b}}
$$

This gives a value of $45 \AA$, which is in good agreement with the actual value ${ }^{28}$ of $25 \AA$.

In order to evaluate the effect of quantum confinement separately on both the conduction band (CB) and the valence band (VB), we have aligned the bulk density of states (DOS) with that of the QD using an estimated offset between the valence band of bulk $\mathrm{MAPbI}_{3}$ and the vacuum level of the QD. ${ }^{30}$ One can see in Figure 1(b) that the offset with respect to the bulk CB and VB is symmetric which is attributed to the equal effective hole and electron masses. Indeed, previous $\mathrm{DFT}^{6,31}$ calculations show 
that this is the case.

Previous experiments ${ }^{32,33}$ show significant differences in the Urbach tails of mesoporous and planar perovskite films. It was indicated that in mesoporous films the Urbach tail is on the order of 100-200 meV ${ }^{32}$ whereas in the case of planar films ${ }^{33}$ the Urbach tail on the order of $15 \mathrm{meV}$. To shed some light on why there is such a discrepancy in the DOS of subgap tails, we have carried out a calculation of the local density of states (LDOS) separated into surface atomic and core atomic regions for the QD and planar film with net zero dipole moment. Based on Figure 1(c), one can see that there are surface associated states lying $0.1 \mathrm{eV}$ below the conduction band edge of the core-like region, thus suggesting that the Urbach tail found in mesoporous films could be associated with the surface - like states found in QDs. To analyze this more closely, we carried out multiple calculations on various sizes of QDs and still found that the offset between surface like and core-like states in CB or VB (see Supporting Information) lies in between 100-200 meV. As mentioned previously, the Urbach tail in the planar films is $15 \mathrm{meV}$ which is in contrast to that of QDs. Our results indicate this is indeed the case, as shown in Figure 1(d), where the surface LDOS and core-like LDOS have exactly the same onset on both CB and VB, respectively.

Conventional Ab Initio molecular dynamics (MD) is usually performed within a few to a few tens of picoseconds for most typical structures (1-200 atoms) and mostly performed to accumulate average statistical quantities such as vibrational density of states along with an IR and Raman spectrum. Replica exchange molecular dynamics (REMD) ${ }^{34,35}$ or Parallel tempering, ${ }^{36}$ where instead of MD one exploits Monte-Carlo (MC) between replica hops, is on the other hand a very powerful method which outperforms simulated annealing ${ }^{36}$ in searching for global minima structures and also allows enhanced phase space sampling for many structures. Typical REMD simulations consist of $\mathrm{N}$ independent parallel copies of the original system (replicas) being run at conventional MD level in the canonical ensemble for a specified number of steps at M different temperatures, indicated as "MD evolution" in Figure 2. In order to guarantee a random temperature walk and thus enhanced canonical ensemble sampling, one has to exchange replicas as shown in Figure 2 between "MD evolution" steps based on the Metropolis criterion. ${ }^{37}$ These swaps happen between replica temperatures based on exchange probability from replica $\mathrm{X}$ to neighboring replica $\mathrm{X}^{\prime}$ as defined: 


$$
w\left(X \rightarrow X^{\prime}\right)= \begin{cases}\exp (-\Delta) & \text { if } \Delta>0 \\ 1 & \text { if } \Delta \leq 0\end{cases}
$$

where $\Delta$ is defined as:

$$
\Delta=\left[\beta_{n}-\beta_{m}\right]\left(E(\mathbf{q})_{(m)}-E(\mathbf{q})_{(n)}\right)
$$

where $\beta_{n}$ and $\beta_{m}$ are $1 / \mathrm{kT}_{n}$ and $1 / \mathrm{kT}_{m}, E(\mathbf{q})_{(m)}$ and $E(\mathbf{q})_{(n)}$ are the potential energies at neighboring temperature replicas $\mathrm{T}_{n}$ and $\mathrm{T}_{m}$, such that $\mathrm{T}_{m}>\mathrm{T}_{n}$, and $\mathbf{q}_{m}$ and $\mathbf{q}_{n}$ are the generalized coordinates.

So far REMD ${ }^{34}$ has been limited to the classical MD calculations; however, some attempts have been made to utilize it in the context of pure quantum mechanical calculations ${ }^{35,38}$ for small clusters and intermediate-sized structures in the regime of coupled QM/MM simulations. ${ }^{39,40}$ We have implemented the REMD algorithm on top of CP2K ${ }^{41}$ within Born-Oppenheimer MD. The algorithmic details can be found elsewhere. ${ }^{34,35}$

First, we looked at the energy landscape searching properties of REMD for the example of the offstoichiometric dot $\left(\mathrm{MA}_{27} \mathrm{~Pb}_{7} \mathrm{I}_{35}\right)$. Figure 3(a) shows the potential energy of the off-stoichiometric dot as a function of the MD (REMD) steps. One can see that REMD at $300 \mathrm{~K}$ finds a much lower energy state in a very short time compared to conventional MD, thus locating much deeper energy minima (see Supporting Information Figure S3). It should be noted that at these timescales (ps) it is still too far from accessing all energetically possible configurations or, in other words, completely sampling the canonical ensemble. However, even at these timescales REMD outperforms conventional MD in locating energetically favourable configurations. On the other hand, to study the effect of enhanced sampling one has to sustain the potential energy overlap between replicas at neighboring temperatures such that one achieves the random temperature walk of the replica. Figure 3(b) shows the potential energy of the replicas at $300 \mathrm{~K}$ and $500 \mathrm{~K}$ for both conventional MD and REMD simulations as a function of MD (REMD) steps. As can be seen, there is no gain in the energy of REMD compared to the conventional MD. However, by looking at Figure 3(c) one can see that potential energy overlap is sustained over the course of the REMD simulation, resulting in the random temperature walk of replicas as demonstrated for the example of a replica at $300 \mathrm{~K}$ as shown in Figure 3(d). Thus, in the case of the 
stoichiometric dots the absence of energy gain could be attributed to the existence of a large number of energetic minima associated with many possible MA orientations which vary within few kT. Finally, to demonstrate that REMD indeed samples more configurations within a shorter time, we plotted a normalized 2D distribution of MA angles, defined as $\mathrm{C}-\mathrm{N}$ bond angle with respect to the cubic unit cell axis $(\theta, \phi)$, for the MD and REMD replica at $300 \mathrm{~K}$. Figure 4 shows that the preferential alignment of MA axis is along the angles $\phi=0$ and $\theta=0,90,180,270$ degrees, which indicates facial alignment of the MA cation and is in agreement with previous calculations of bulk MA distribution in the cubic perovskite phase. ${ }^{42}$ In addition, one can see from Figure 4 that there is an additional peaked angular distribution along the $\phi=70$ degrees which comes from the MA cations located in the surface region. To compare how efficient REMD is compared to conventional MD calculations, we have plotted distribution of MA angles accumulated at different times. By looking at Figures 4(a), 4(b) and Figures 4(c) one can see that, in the case of the REMD calculations, many more MA angular configurations are sampled with respect to the conventional MD calculations. More specifically, black regions, highlighted by yellow polygons, in Figure 4(a) and 4(b) indicate regions of MA orientations which are unsampled during MD simulation, whereas in the case of the REMD calculations (Figure 4(c)) it is seen that these regions are much better sampled. Thus, the usage of REMD calculations within mesoscopic scales opens new possibilities to study molecular systems at a longer time scales comparable to classical MD simulations.

In conclusion, we have computationally investigated a novel materials system - organolead halide perovskite quantum dots. It is shown that stoichiometric perovskite QDs are free of deep traps. The effect of quantum confinement is demonstrated and an estimate for the upper bound of the Bohr exciton is given. It is demonstrated that both the $\mathrm{CB}$ and $\mathrm{VB}$ participate in quantum confinement, which is in line with the ambipolar perovskite behaviour. In addition, an explanation is given as to why there is such a discrepancy in the Urbach tail seen in the mesoporous and planar perovskite films. Finally, replica exchange molecular dynamics (REMD) has been implemented within DFT for realistically sized systems for the first time. It is shown that REMD compares favourably to conventional Ab Initio MD in locating deep lying minima structures and at the same time sampling of the phase space is enhanced based on the analysis of the 2D MA angular distribution. 


\section{Methods}

Calculations were performed within the DFT formalism using the Perdew-Burke-Ernzerhof $(\mathrm{PBE})^{43}$ GGA exchange correlation functional. All calculations were performed utilizing the CP2K ${ }^{41}$ package within Gaussian-augmented plane waves (GAPW) dual basis set using the molecularly optimized MOLOPT $^{41}$ double $\zeta$-valence polarized (mDZVP) basis set implemented in CP2K code which has very small BSSE errors in gas and condensed phases. ${ }^{4,44-47}$ The plane-wave cutoff was 300 Ry, which is suitable for the Goedecker-Teter-Hutter pseudopotentials. ${ }^{48}$ Spin polarized (LSDA) and spin-unpolarized caculations (LDA) were performed in the case of the odd-even number of electrons. The initial structural minimization was performed with the help of the Broyden-Fletcher-Goldfarb-Shanno algorithm ${ }^{49}$ (BFGS). Initial structural optimiziation was considered converged if the forces acting on the atoms were less than $0.04 \mathrm{eV} \cdot \AA^{-1}$.

In order to construct quantum dots both stoichiometric and off-stoichiometric, we have cut them from cubic $(\mathrm{a}=6.3 \AA) \mathrm{MAPbI}_{3}$ perovskite phase. Stoichiometric and off-stoichiometric dots were constructed as cuboids with terminated facets $([100],[010],[001])$ while preserving bulk stoichiometry I:Pb:MA as 3:1:1 in the former case (asymmetric termination $\mathrm{MAI}-\mathrm{PbI}_{2}$ ), and having various symmetric terminations $\left(\mathrm{PbI}_{2}-\mathrm{PbI}_{2}\right.$ or MAI-MAI) for the off-stoichiometric dots. After initial construction and optimization, dots were subsequently subjected to ab initio MD at various temperatures (100 K-600 K) for 20 ps with subsequent monitoring of the bandgap through the course of MD simulations. Various configurations (1020) for both stoichiometric and off-stoichiometric dots were modeled through MD and then geometrically optimized via the BFGS algorithm. The configurations possessing the lowest energy were selected. This procedure helped tremendously in identifying trap-free structures in the case of the stoichiometric dots; however, it had little or no effect on curing traps in the case of off-stoichiometric dots. The best configurations were subsequently fed into MD and REMD production simulations. Radii of the particles were estimated via radius of gyration $\mathrm{R}_{g}^{2}=\frac{3}{5} \mathrm{R}^{2}$ averaged over molecular dynamics. Vacuum boundary conditions were applied with a vacuum box of $50 \AA$. We performed the Born-Oppenheimer molecular dynamics (BOMD) in NVT ensemble using Nose-Hoover thermostat with 4 chains and with 0.5 fs time step and 100 fs time constant over 50 ps with initial 10 ps taken as equilibration time. Replica exchange molecular dynamics (REMD) was implemented on top of CP2K code and swap attempts were tried 
every 50 MD steps. REMD calculations were carried over 5-20 ps total time. Total number of replicas varied between 14-20 for the temperature range of 200-600K.

\section{Supporting Information.}

Supporting Information Available: Additional figures for the off-stoichiometric dots and figures showing core-like and surfacec-like states for all stoichiometric dots. This material is available free of charge via the Internet at http://pubs.acs.org.

\section{Acknowledgments}

This publication is based in part on work supported by Award KUS-11-009-21, made by King Abdullah University of Science and Technology (KAUST), by the Ontario Research Fund Research Excellence Program, and by the Natural Sciences and Engineering Research Council (NSERC) of Canada. Computations were performed on the Southern Ontario Smart Computing Innovation Platform (SOSCIP) Blue Gene/Q supercomputer located at the University of Toronto's SciNet ${ }^{50}$ HPC facility. The SOSCIP multi-university/industry consortium is funded by the Ontario Government and the Federal SciNet is funded by: the Canada Foundation for Innovation under the auspices of Compute Canada; the Government of Ontario; Ontario Research Fund - Research Excellence; and the University of Toronto. 


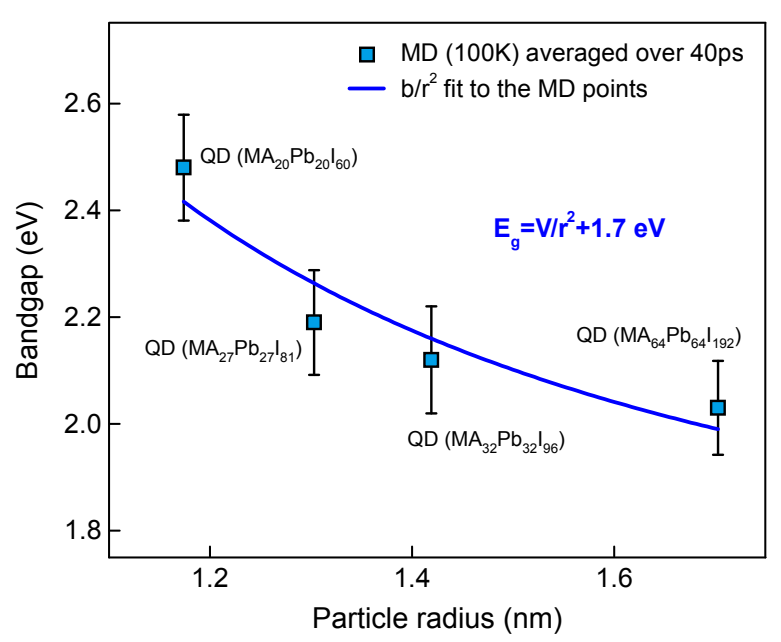

(a)

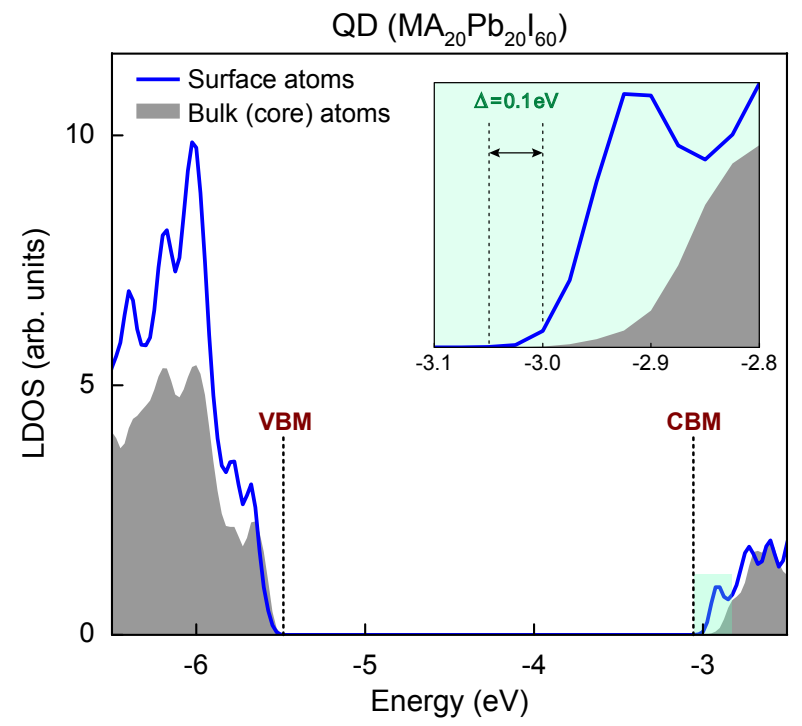

(c)

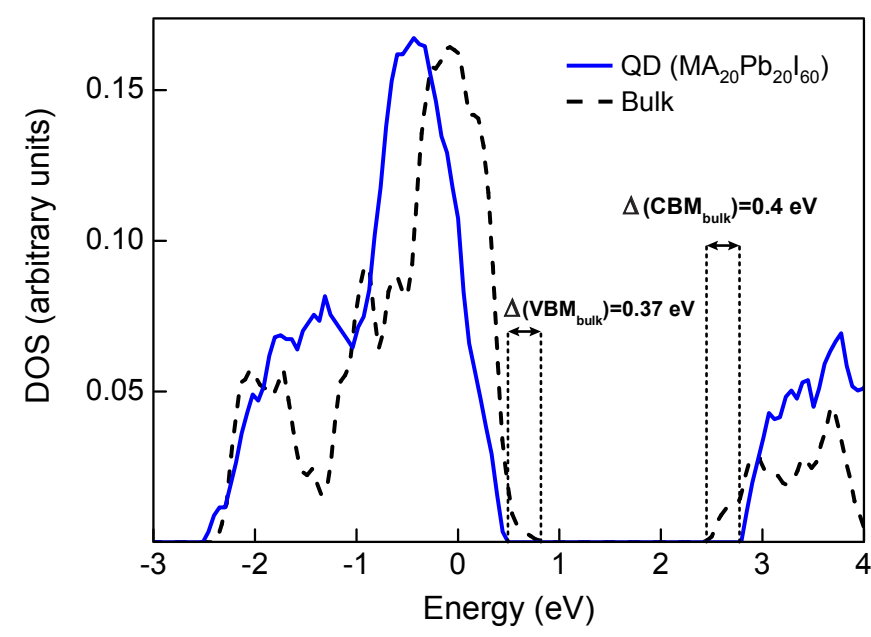

(b)

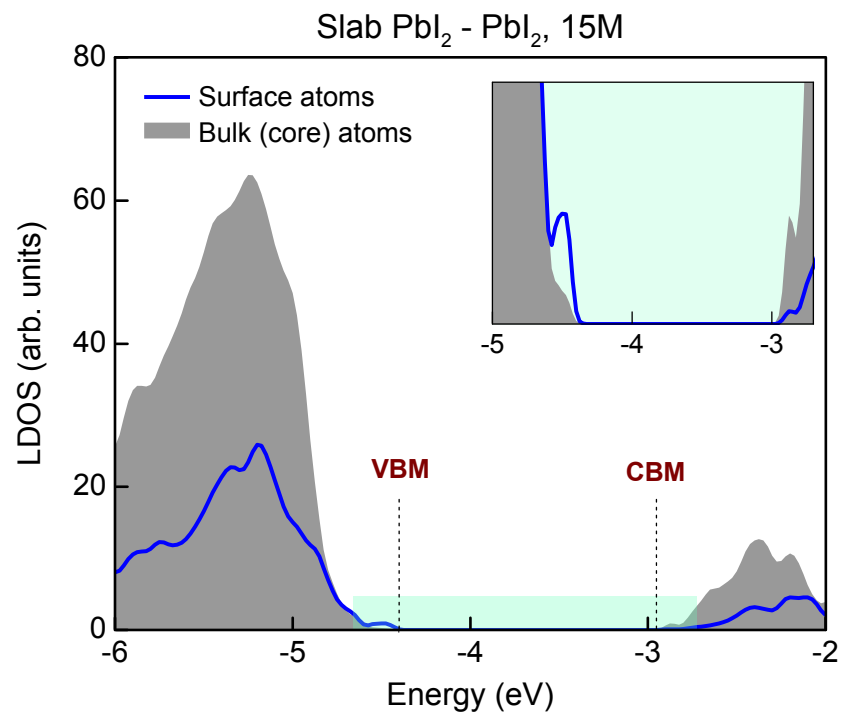

(d)

Figure 1: (a) Bandgap variation over QD radius, $b=100 \mathrm{eV}^{*} \AA^{2}$ (b) Deep Pb-level aligned Bulk $\mathrm{MAPbI}_{3}$ and $\mathrm{QD}\left(\mathrm{MA}_{20} \mathrm{~Pb}_{20} \mathrm{I}_{60}\right)$ showing that the confinement comes from conduction and valence bands. Local density of states resolved into the Surface DOS and Bulk DOS for (c) the QD $\left(\mathrm{MA}_{20} \mathrm{~Pb}_{20} \mathrm{I}_{60}\right)$ and (d) Symmetric - off-stoichiometric slab terminated with $\mathrm{PbI}_{2}$ on both ends, with net zero dipole moment along z-axis. 


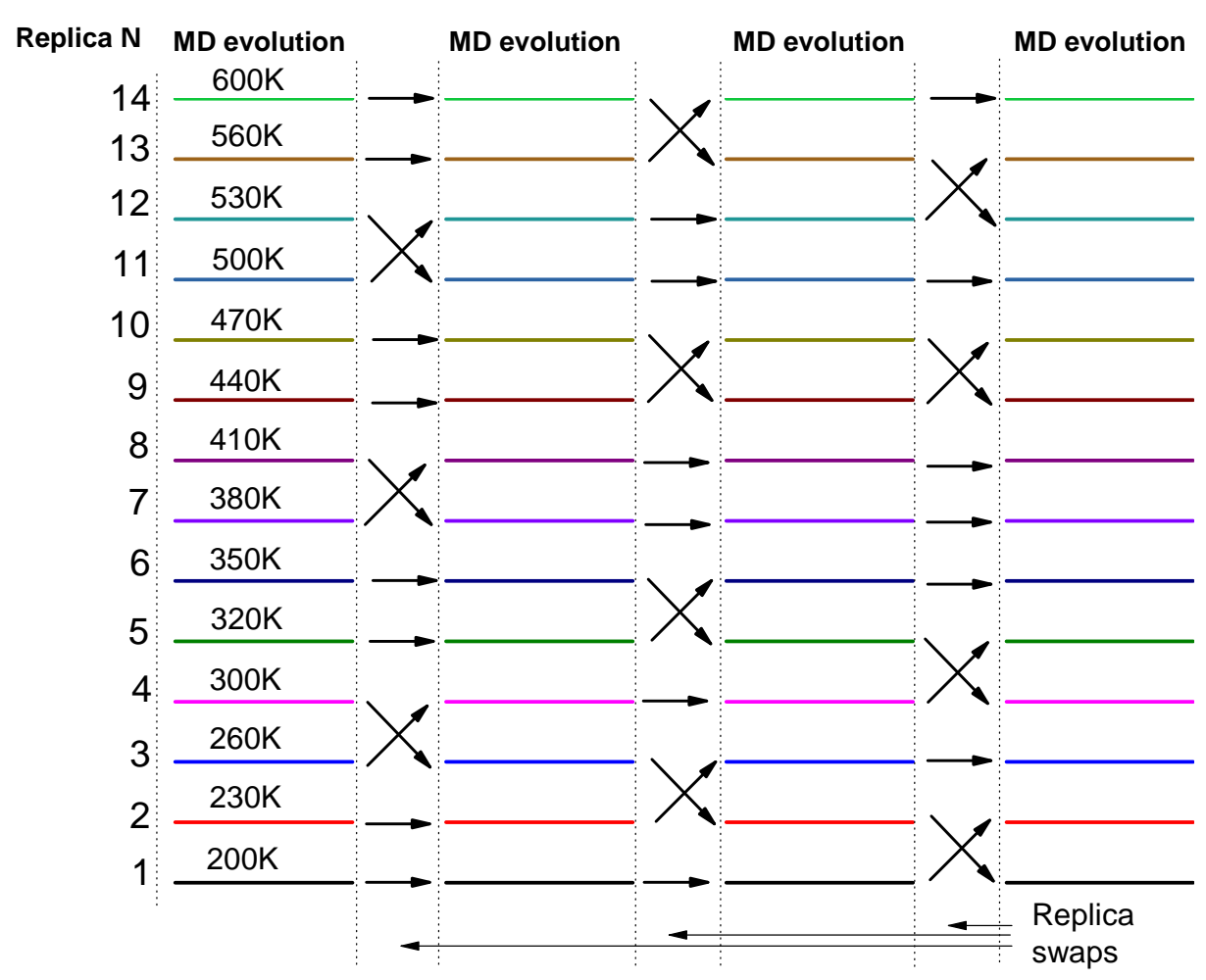

Figure 2: REMD schematics showing the REMD algorithm 


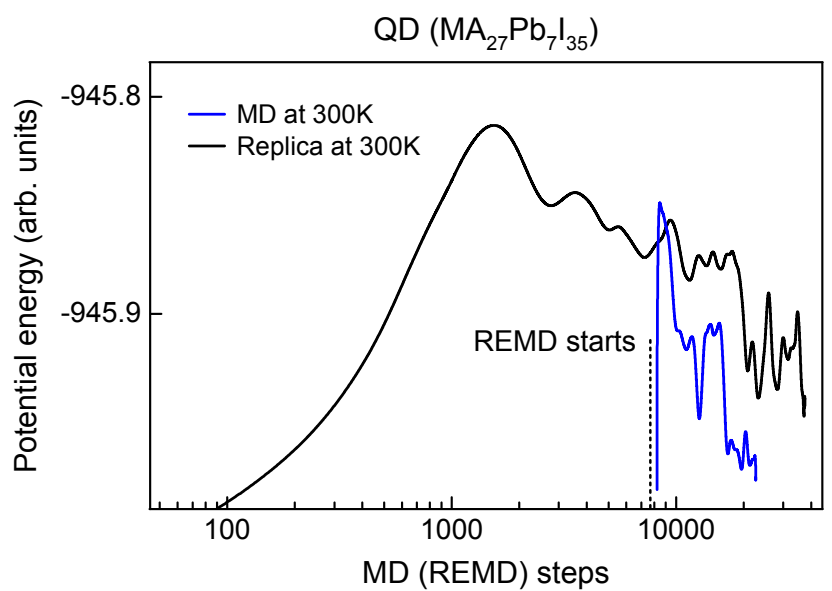

(a)

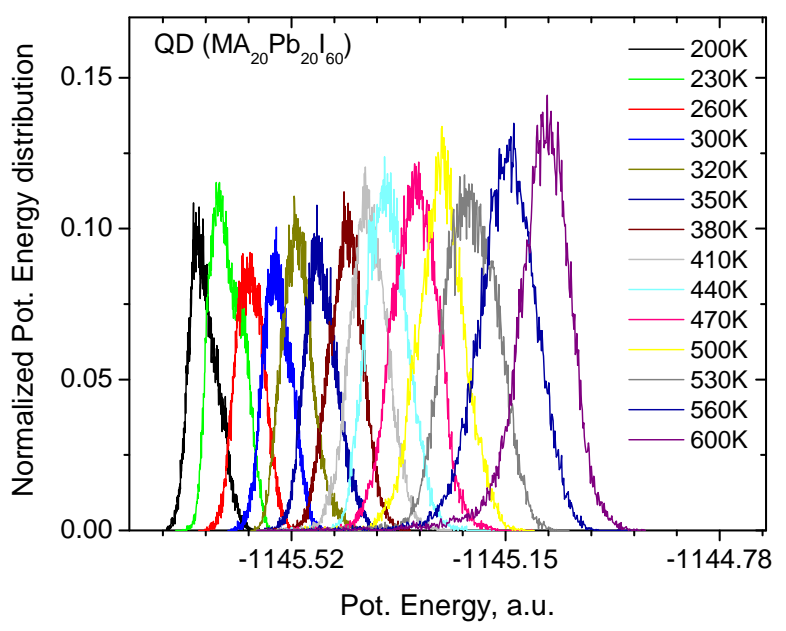

(c)

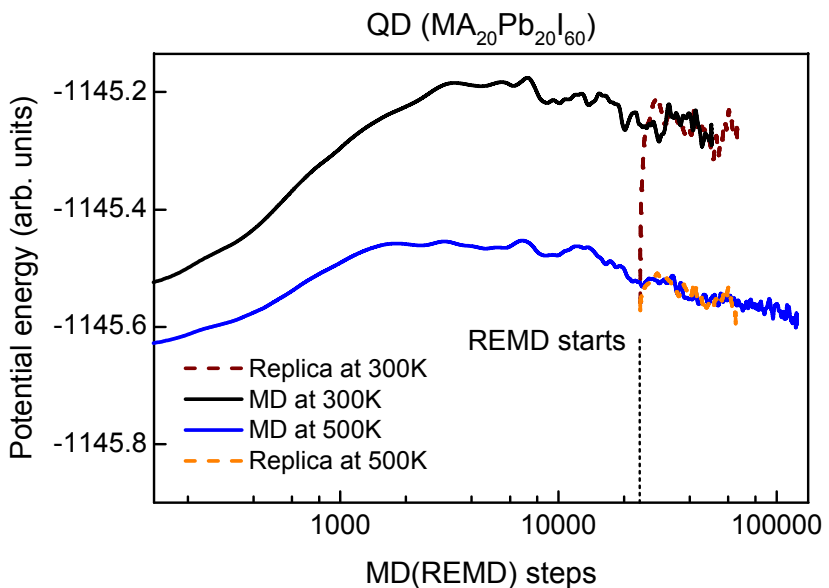

(b)

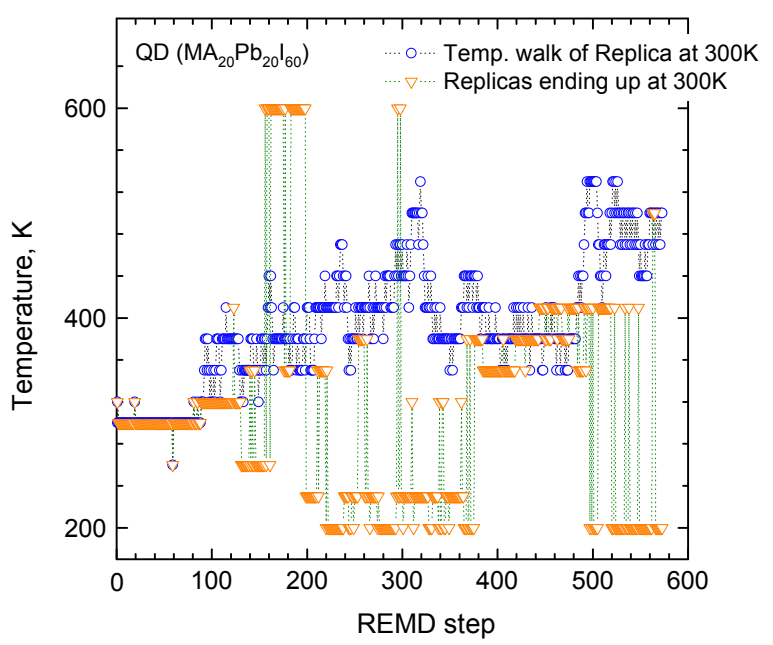

(d)

Figure 3: Characterization of the REMD algorithm implementation (a) Energy landscape searching for the off-stoichiometric dot $\mathrm{MA}_{27} \mathrm{~Pb}_{7} \mathrm{I}_{35}$, showing that REMD finds much lower energy structures (b) Energy of the $\mathrm{MA}_{20} \mathrm{~Pb}_{20} \mathrm{I}_{60}$ dot vs. MD step in REMD and conventional MD calculations showing that energetically REMD and MD are similar in case of stoichiometric dots (c) Potential energy overlap for replicas at various temperatures (d) Random temperature walk of Replica, initially started at $300 \mathrm{~K}$ and Replicas at different temperatures that end up in equilibration at $300 \mathrm{~K}$. Please note that in the case of (a) and (b) log scale for $\mathrm{X}$ axis is used for clarity. 

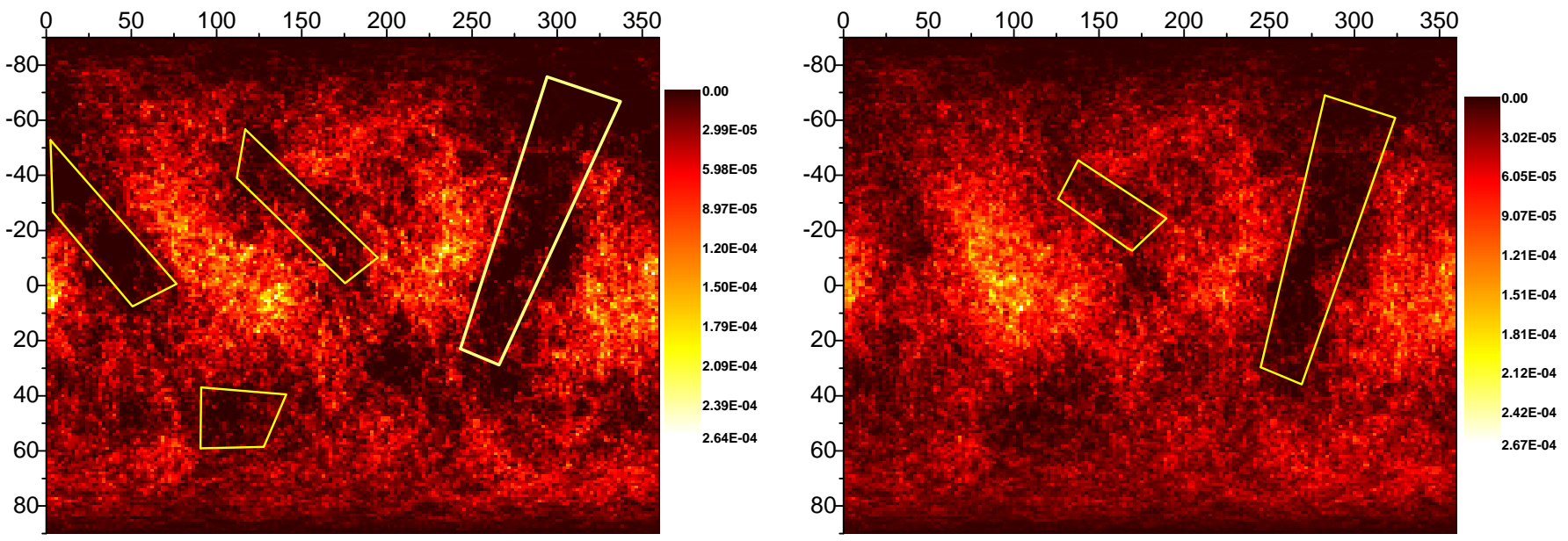

(a)

(b)

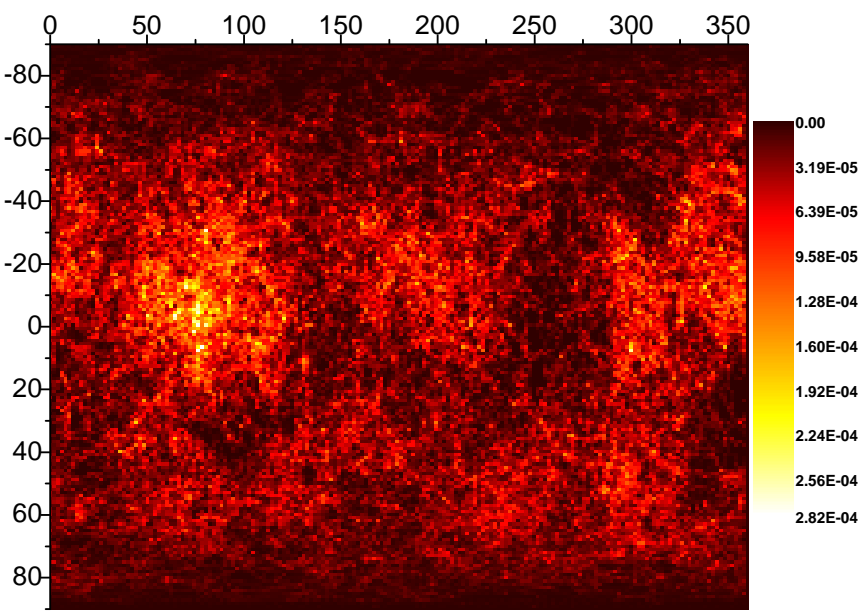

(c)

Figure 4: Normalized 2D maps of MA orientation $(\mathrm{X}-\theta, \mathrm{Y}-\phi)$ in case of (a) conventional MD at $300 \mathrm{~K}$ for 26 ps and (b) 55 ps and (c) REMD at $300 \mathrm{~K}$ for 16 ps. 


\section{References}

(1) Kim, H.-S.; Lee, C.-R.; Im, J.-H.; Lee, K.-B.; Moehl, T.; Marchioro, A.; Moon, S.-J.; HumphryBaker, R.; Yum, J.-H.; Moser, J. E.; et al., Lead Iodide Perovskite Sensitized All-Solid-State Submicron Thin Film Mesoscopic Solar Cell with Efficiency Exceeding 9\%. Sci. Rep. 2012, 2, 591.

(2) Lee, M. M.; Teuscher, J.; Miyasaka, T.; Murakami, T. N.; Snaith, H. J. Efficient Hybrid Solar Cells Based on Meso-Superstructured Organometal Halide Perovskites. Science 2012, 338, 643-647.

(3) Burschka, J.; Pellet, N.; Moon, S.-J.; Humphry-Baker, R.; Gao, P.; Nazeeruddin, M. K.; Grätzel, M. Sequential Deposition as a Route to High-Performance Perovskite-Sensitized Solar Cells. Nature 2013, 499, 316-319.

(4) Buin, A.; Pietsch, P.; Xu, J.; Voznyy, O.; Ip, A. H.; Comin, R.; Sargent, E. H. Materials Processing Routes to Trap-Free Halide Perovskites. Nano Lett. 2014, 14, 6281-6286.

(5) Kim, J.; Lee, S.-H.; Lee, J. H.; Hong, K.-H. The Role of Intrinsic Defects in Methylammonium Lead Iodide Perovskite. J. Phys. Chem. Lett. 2014, 5, 1312-1317.

(6) Menéndez-Proupin, E.; Palacios, P.; Wahnón, P.; Conesa, J. C. Self-Consistent Relativistic Band Structure of the $\mathrm{CH}_{3} \mathrm{NH}_{3} \mathrm{PbI}_{3}$ Perovskite. Phys. Rev. B 2014, 90, 045207.

(7) Miller, E. M.; Zhao, Y.; Mercado, C.; Saha, S.; Luther, J. M.; Zhu, K.; Stevanovic, V.; Perkins, C. L.; van de Lagemaat, J. Substrate-Controlled Band Positions in $\mathrm{CH}_{3} \mathrm{NH}_{3} \mathrm{PbI}_{3}$ Perovskite Films. Phys. Chem. Chem. Phys. 2014, 16, 22122-22130.

(8) Haruyama, J.; Sodeyama, K.; Han, L.; Tateyama, Y. Termination Dependence of Tetragonal $\mathrm{CH}_{3} \mathrm{NH}_{3} \mathrm{PbI}_{3}$ Surfaces for Perovskite Solar Cells. J. Phys. Chem. Lett. 2014, 5, 2903-2909.

(9) Xing, G.; Mathews, N.; Sun, S.; Lim, S. S.; Lam, Y. M.; Grätzel, M.; Mhaisalkar, S.; Sum, T. C. Long-Range Balanced Electron- and Hole-Transport Lengths in Organic-Inorganic $\mathrm{CH}_{3} \mathrm{NH}_{3} \mathrm{PbI}_{3}$. Science 2013, 342, 344-347.

(10) Yin, W.-J.; Shi, T.; Yan, Y. Unique Properties of Halide Perovskites as Possible Origins of the Superior Solar Cell Performance. Adv. Mat. 2014, 26, 4653-4658. 
(11) Yin, W.-J.; Shi, T.; Yan, Y. Unusual Defect Physics in $\mathrm{CH}_{3} \mathrm{NH}_{3} \mathrm{PbI}_{3}$ Perovskite Solar Cell Absorber. Appl. Phys. Lett. 2014, 10, 063903.

(12) Stranks, S. D.; Eperon, G. E.; Grancini, G.; Menelaou, C.; Alcocer, M. J. P.; Leijtens, T.; Herz, L. M.; Petrozza, A.; Snaith, H. J. Electron-Hole Diffusion Lengths Exceeding 1 Micrometer in an Organometal Trihalide Perovskite Absorber. Science 2013, 342, 341-344.

(13) Shkrob, I. A.; Marin, T. W. Charge Trapping in Photovoltaically Active Perovskites and Related Halogenoplumbate Compounds. J. Phys. Chem. Lett. 2014, 5, 1066-1071.

(14) Schmidt, L. C.; Pertegs, A.; Gonzlez-Carrero, S.; Malinkiewicz, O.; Agouram, S.; Mnguez Espallargas, G.; Bolink, H. J.; Galian, R. E.; Prez-Prieto, J. Nontemplate Synthesis of $\mathrm{CH}_{3} \mathrm{NH}_{3} \mathrm{PbBr}_{3}$ Perovskite Nanoparticles. J. Am. Chem. Soc. 2014, 136, 850-853.

(15) Heo, J. H.; Song, D. H.; Im, S. H. Planar $\mathrm{CH}_{3} \mathrm{NH}_{3} \mathrm{PbBr}_{3}$ Hybrid Solar Cells with 10.4\% Power Conversion Efficiency, Fabricated by Controlled Crystallization in the Spin-Coating Process. Adv. Mat. 2014, 26, 8179-8183.

(16) Zhang, M.; Yu, H.; Lyu, M.; Wang, Q.; Yun, J.-H.; Wang, L. Composition-Dependent Photoluminescence Intensity and Prolonged Recombination Lifetime of Perovskite $\mathrm{CH}_{3} \mathrm{NH}_{3} \mathrm{PbBr}_{3-x} \mathrm{Cl}_{x}$ films. Chem. Commun. 2014, 50, 11727-11730.

(17) Liu, M.; Johnston, M. B.; Snaith, H. J. Efficient Planar Heterojunction Perovskite Solar Cells by Vapour Deposition. Nature 2013, 501, 395-398.

(18) Protesescu, L.; Yakunin, S.; Bodnarchuk, M. I.; Krieg, F.; Caputo, R.; Hendon, C. H.; Yang, R. X.; Walsh, A.; Kovalenko, M. V. Nanocrystals of Cesium Lead Halide Perovskites $\left(\mathrm{CsPbX}_{3}, \mathrm{X}=\mathrm{Cl}\right.$, Br, and I): Novel Optoelectronic Materials Showing Bright Emission with Wide Color Gamut. Nano Lett. ASAP.

(19) Di, D.; Musselman, K. P.; Li, G.; Sadhanala, A.; Ievskaya, Y.; Song, Q.; Tan, Z.-K.; Lai, M. L.; MacManus-Driscoll, J. L.; Greenham, N. C.; Friend, R. H. Size-Dependent Photon Emission from Organometal Halide Perovskite Nanocrystals Embedded in an Organic Matrix. J. Phys. Chem. Lett. 2015, 6, 446-450. 
(20) Park, B.-W.; Philippe, B.; Gustafsson, T.; Sveinbjrnsson, K.; Hagfeldt, A.; Johansson, E. M. J.; Boschloo, G. Enhanced Crystallinity in Organic-Inorganic Lead Halide Perovskites on Mesoporous $\mathrm{TiO}_{2}$ via Disorder-Order Phase Transition. Chem. Mater. 2014, 26, 4466-4471.

(21) Horvth, E.; Spina, M.; Szekrnyes, Z.; Kamars, K.; Gaal, R.; Gachet, D.; Forr, L. Nanowires of Methylammonium Lead Iodide $\left(\mathrm{CH}_{3} \mathrm{NH}_{3} \mathrm{PbI}_{3}\right)$ Prepared by Low Temperature Solution-Mediated Crystallization. Nano Lett. 2014, 14, 6761-6766.

(22) Pistor, P.; Borchert, J.; Franzel, W.; Csuk, R.; Scheer, R. Monitoring the Phase Formation of Coevaporated Lead Halide Perovskite Thin Films by in Situ X-ray Diffraction. J. Phys. Chem. Lett. 2014, 5, 3308-3312.

(23) Naicker, P. K.; Cummings, P. T.; Zhang, H.; Banfield, J. F. Characterization of Titanium Dioxide Nanoparticles Using Molecular Dynamics Simulations. J. Phys. Chem. B 2005, 109, 15243-15249.

(24) Kim, D.; Kim, D.-H.; Lee, J.-H.; Grossman, J. C. Impact of Stoichiometry on the Electronic Structure of PbS Quantum Dots. Phys. Rev. Lett. 2013, 110, 196802.

(25) Voznyy, O.; Thon, S. M.; Ip, A. H.; Sargent, E. H. Dynamic Trap Formation and Elimination in Colloidal Quantum Dots. J. Phys. Chem. Lett. 2013, 4, 987-992.

(26) Leijtens, T.; Stranks, S. D.; Eperon, G. E.; Lindblad, R.; Johansson, E. M. J.; McPherson, I. J.; Rensmo, H.; Ball, J. M.; Lee, M. M.; Snaith, H. J. Electronic Properties of Meso-Superstructured and Planar Organometal Halide Perovskite Films: Charge Trapping, Photodoping, and Carrier Mobility. ACS Nano 2014, 8, 7147-7155.

(27) Bretschneider, S. A.; Weickert, J.; Dorman, J. A.; Schmidt-Mende, L. Research Update: Physical and electrical characteristics of lead halide perovskites for solar cell applications. APL Mat. 2014, 2, 040701.

(28) Ma, J.; Wang, L.-W. Nanoscale Charge Localization Induced by Random Orientations of Organic Molecules in Hybrid Perovskite $\mathrm{CH}_{3} \mathrm{NH}_{3} \mathrm{PbI}_{3}$. Nano Lett. 2015, 15, 248-253.

(29) Einevoll, G. T. Confinement of Excitons in Quantum Dots. Phys. Rev. B 1992, 45, 3410-3417. 
(30) Even, J.; Pedesseau, L.; Jancu, J.-M.; Katan, C. Importance of Spin-Orbit Coupling in Hybrid Organic/Inorganic Perovskites for Photovoltaic Applications. J. Phys. Chem. Lett. 2013, 4, 29993005 .

(31) Giorgi, G.; Fujisawa, J.-I.; Segawa, H.; Yamashita, K. Small Photocarrier Effective Masses Featuring Ambipolar Transport in Methylammonium Lead Iodide Perovskite: A Density Functional Analysis. J. Phys. Chem. Lett. 2013, 4, 4213-4216.

(32) Yamada, Y.; Nakamura, T.; Endo, M.; Wakamiya, A.; Kanemitsu, Y. Near-Band-Edge Optical Responses of Solution-Processed Organic-Inorganic Hybrid Perovskite $\mathrm{CH}_{3} \mathrm{NH}_{3} \mathrm{PbI}_{3}$ on Mesoporous $\mathrm{TiO}_{2}$ Electrodes. Appl. Phys. Expr. 2014, 7, 032302.

(33) De Wolf, S.; Holovsky, J.; Moon, S.-J.; Lper, P.; Niesen, B.; Ledinsky, M.; Haug, F.-J.; Yum, J.-H.; Ballif, C. Organometallic Halide Perovskites: Sharp Optical Absorption Edge and Its Relation to Photovoltaic Performance. J. Phys. Chem. Lett. 2014, 5, 1035-1039.

(34) Sugita, Y.; Okamoto, Y. Replica-Exchange Molecular Dynamics Method for Protein Folding. Chem. Phys. Lett. 1999, 314, $141-151$.

(35) Ishikawa, Y.; Sugita, Y.; Nishikawa, T.; Okamoto, Y. Ab Initio Replica-Exchange Monte Carlo Method for Cluster Studies. Chem. Phys. Lett. 2001, 333, 199 - 206.

(36) Earl, D. J.; Deem, M. W. Parallel Tempering: Theory, Applications, and New Perspectives. Phys. Chem. Chem. Phys. 2005, 7, 3910-3916.

(37) Sugita, Y.; Kitao, A.; Okamoto, Y. Multidimensional Replica-Exchange Method for Free-Energy Calculations. J. Chem. Phys. 2000, 113, 6042-6051.

(38) Li, H.; Yang, W. Sampling Enhancement for the Quantum Mechanical Potential Based Molecular Dynamics Simulations: A General Algorithm and its Extension for Free Energy Calculation on Rugged Energy Surface. J. Chem. Phys. 2007, 126, 114104.

(39) Buin, A.; Wang, H.; Consta, S.; Huang, Y. A Study of Conformational Equilibrium of 1,1,2Trichloroethane in FAU-Type Zeolites. Microporous Mesoporous Mater. 2014, 183, 207 - 217. 
(40) Buin, A.; Ma, J.; Huang, Y.; Consta, S.; Hui, Z. Conformational Changes of trans-1,2Dichlorocyclohexane Adsorbed in Zeolites Studied by FT-Raman Spectroscopy and Molecular QM/MM Simulations. J. Phys. Chem. C 2012, 116, 8608-8618.

(41) VandeVondele, J.; Hutter, J. Gaussian Basis Sets for Accurate Calculations on Molecular Systems in Gas and Condensed Phases. J. Chem. Phys. 2007, 127, 114105.

(42) Frost, J. M.; Butler, K. T.; Walsh, A. Molecular Ferroelectric Contributions to Anomalous Hysteresis in Hybrid Perovskite Solar Cells. APL Mat. 2014, 2, 081506.

(43) Perdew, J. P.; Burke, K.; Ernzerhof, M. Generalized Gradient Approximation Made Simple. Phys. Rev. Lett. 1996, 77, 3865-3868.

(44) Leitsmann, R.; Bhm, O.; Plnitz, P.; Radehaus, C.; Schaller, M.; Schreiber, M. Adsorption Mechanisms of Fluorocarbon Polymers at Ultra Low-k Surfaces. Surf. Sci. 2010, 604, 1808 - 1812.

(45) Takaluoma, T. T.; Laasonen, K.; Laitinen, R. S. Molecular Dynamics Simulation of the Solid-State Topochemical Polymerization of $\mathrm{S}_{2} \mathrm{~N}_{2}$. Inorg. Chem. 2013, 52, 4648-4657.

(46) Bork, N.; Loukonen, V.; Vehkamki, H. Reactions and Reaction Rate of Atmospheric $\mathrm{SO}_{2}$ and $\mathrm{O}_{3}$ $\left(\mathrm{H}_{2} \mathrm{O}\right)_{n}$ Collisions via Molecular Dynamics Simulations. J. Phys. Chem. A 2013, 117, 3143-3148.

(47) Smecca, E.; Motta, A.; Fragal, M. E.; Aleeva, Y.; Condorelli, G. G. Spectroscopic and Theoretical Study of the Grafting Modes of Phosphonic Acids on ZnO Nanorods. J. Phys. Chem. C 2013, 117, 5364-5372.

(48) Hartwigsen, C.; Goedecker, S.; Hutter, J. Relativistic Separable Dual-Space Gaussian Pseudopotentials from H to Rn. Phys. Rev. B 1998, 58, 3641-3662.

(49) Press, W. H.; Teukolsky, S. A.; Vetterling, W. T.; Flannery, B. P. Numerical Recipes 3rd Edition: The Art of Scientific Computing, 3rd ed.; Cambridge University Press: New York, NY, USA, 2007.

(50) Loken, C.; Gruner, D.; Groer, L.; Peltier, R.; Bunn, N.; Craig, M.; Henriques, T.; Dempsey, J.; Yu, C.-H.; Chen, J.; Dursi, L. J.; et al., SciNet: Lessons Learned from Building a Power-efficient Top-20 System and Data Centre. J. Phys. : Conference Series 2010, 256, 012026. 


\section{TOC Figure}

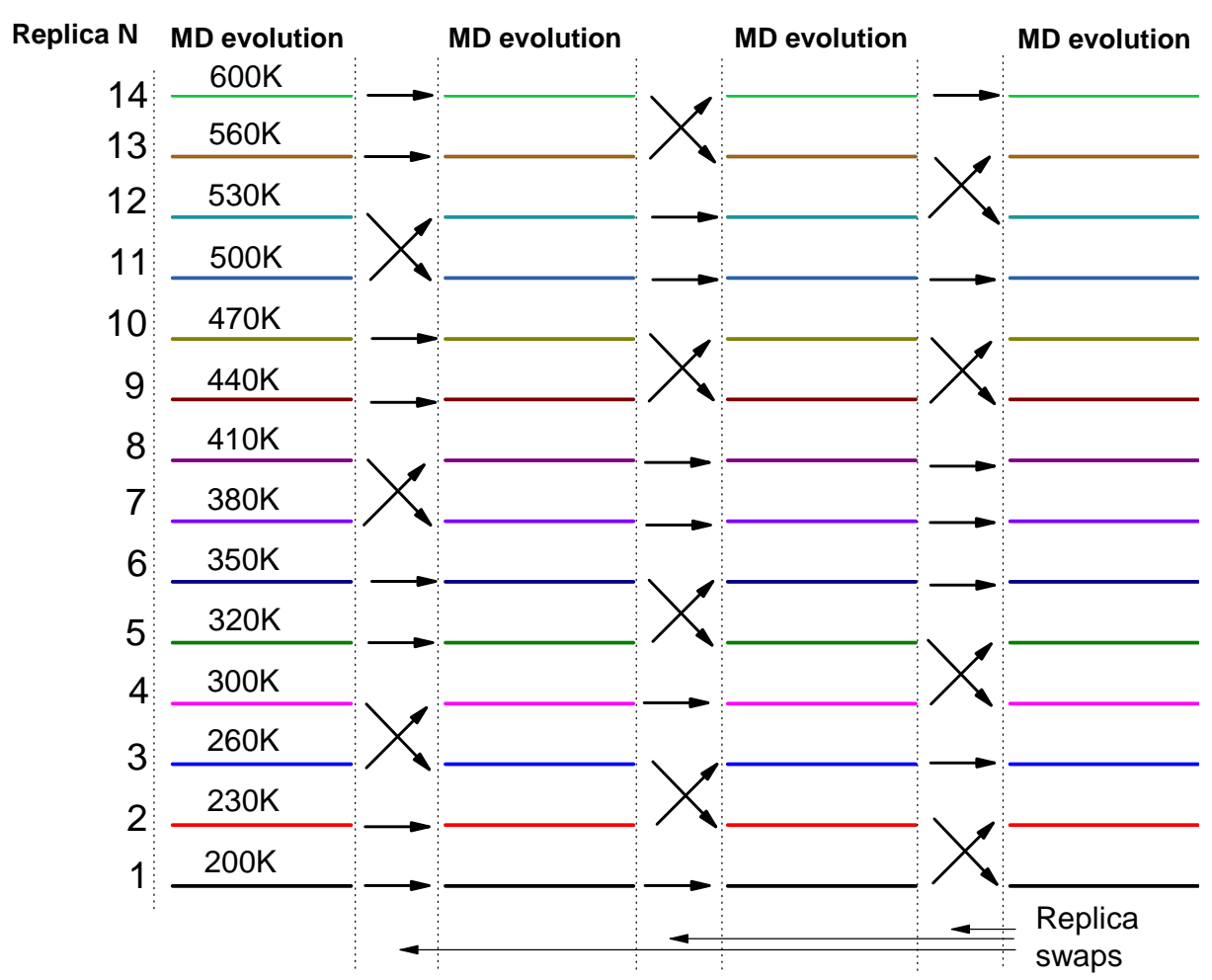

